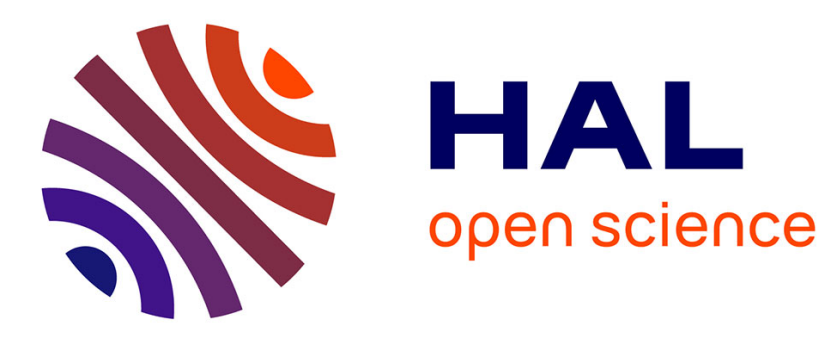

\title{
Flatness of two-input control-affine systems linearizable via a two-fold prolongation
}

Florentina Nicolau, Witold Respondek

\section{To cite this version:}

Florentina Nicolau, Witold Respondek. Flatness of two-input control-affine systems linearizable via a two-fold prolongation. 55th IEEE Conference on Decision and Control, Dec 2016, Las Vegas, United States. hal-01351615

\section{HAL Id: hal-01351615 https://hal.science/hal-01351615}

Submitted on 8 Aug 2016

HAL is a multi-disciplinary open access archive for the deposit and dissemination of scientific research documents, whether they are published or not. The documents may come from teaching and research institutions in France or abroad, or from public or private research centers.
L'archive ouverte pluridisciplinaire HAL, est destinée au dépôt et à la diffusion de documents scientifiques de niveau recherche, publiés ou non, émanant des établissements d'enseignement et de recherche français ou étrangers, des laboratoires publics ou privés. 


\title{
Flatness of two-input control-affine systems linearizable via a two-fold prolongation
}

\author{
Florentina Nicolau ${ }^{a}$ and Witold Respondek ${ }^{b}$
}

\begin{abstract}
We study flatness of two-input control-affine systems, defined on an $n$-dimensional state-space. We give a geometric characterization of systems that become static feedback linearizable after a two-fold prolongation of a suitably chosen control. They form a particular class of flat systems: they are of differential weight $n+4$. We present a normal form compatible with the minimal flat outputs.
\end{abstract}

\section{INTRODUCTION}

In this paper, we study flatness of nonlinear control systems of the form

$$
\Xi: \dot{x}=F(x, u),
$$

where $x$ is the state defined on a open subset $X$ of $\mathbb{R}^{n}$ and $u$ is the control taking values in an open subset $U$ of $\mathbb{R}^{m}$ (more generally, an $n$-dimensional manifold $X$ and an $m$-dimensional manifold $U$, respectively). The dynamics $F$ are smooth and the word smooth will always mean $\mathcal{C}^{\infty}$-smooth.

The notion of flatness has been introduced in control theory in the 1990's, by Fliess, Lévine, Martin and Rouchon [5], [6], see also [10], [11], [13], [21], and has attracted a lot of attention because of its multiple applications in the problem of constructive controllability and motion planning (see, e.g. [7], [22], [25], [20], [14], [23], [24]). Flat systems form a class of control systems whose set of trajectories can be parametrized by $m$ functions and their time-derivatives, $m$ being the number of controls. More precisely, the system $\Xi: \dot{x}=F(x, u)$ is flat if we can find $m$ functions, $\varphi_{i}\left(x, u, \ldots, u^{(l)}\right)$ such that

$$
x=\gamma\left(\varphi, \ldots, \varphi^{(s-1)}\right) \text { and } u=\delta\left(\varphi, \ldots, \varphi^{(s)}\right),
$$

for a certain integer $s$, where $\varphi=\left(\varphi_{1}, \ldots, \varphi_{m}\right)$ is called a flat output. Therefore the time-evolution of all state and control variables can be determined from that of flat outputs without integration and all trajectories of the system can be completely parameterized. A similar notion, of systems of undetermined differential equations integrable without integration, has been studied by Hilbert [8] and Cartan [3], see also [25], where connections between Cartan prolongations and flatness were studied.

Flatness is closely related to the notion of feedback linearization. It is well known that systems linearizable

\footnotetext{
${ }^{a}$ INRIA Sophia Antipolis Mditerrane, 2004 Route des Lucioles, 06902 Valbonne, France, florentina.nicolau, dinria.fr

${ }^{b}$ INSA-Rouen, Laboratoire de Mathématiques, 76801 Saint-Etiennedu-Rouvray, France witold.respondek@insa-rouen. fr
}

via invertible static feedback are flat. Their description (1) uses the minimal possible, which is $n+m$, number of time-derivatives of the components of the flat output $\varphi$. In general, a flat system is not linearizable by static feedback, with the exception of the singleinput case where flatness reduces to static feedback linearization, see [4] and [21]. For any flat system, that is not static feedback linearizable, the minimal number of time-derivatives of $\varphi_{i}$ needed to express $x$ and $u$ (which is called the differential weight [23]) is thus bigger than $n+m$ and measures actually the smallest possible dimension of a precompensator linearizing dynamically the system. The simplest systems for which the differential weight is bigger than $n+m$ are systems linearizable via one-dimensional precompensator (namely one-fold prolongation), thus of differential weight $n+$ $m+1$. That class of systems have been studied in details in [16], [15], see also [17], [18], [19], where the authors presented a complete geometric verifiable characterization, gave normal forms, computed all minimal flat outputs and discussed their uniqueness. The goal of this paper is to generalize those results to control-affine systems that become static feedback linearizable after a two-fold prolongation (thus of differential weight $n+m+2)$. We will consider the case of two-input control systems only. Our aim is to give necessary and sufficient conditions for flatness of differential weight $n+m+2=n+4$ and to describe and understand the geometry of that class of systems. Solving that problem in the simplest case of two controls is interesting for few reasons; firstly, it yields a complete analysis of flatness for a well defined class of systems, secondly, it shows what kind of difficulties one must face when trying to characterize flatness for more general systems (like those requiring more than two prolongations), thirdly, it allows to observe new phenomena like the necessity of distinguishing a special drift.

The paper is organized as follows. In Section II, we recall the definitions of flatness and of differential weight of a flat system as well as the results describing flatness of differential weight $n+3$ for twoinput control-affine systems. In Section III, we give our main results: we characterize control-affine systems that become static feedback linearizable after a twofold prolongation, that is, flat systems of differential weight $n+4$. We also present a normal forms and explain how to compute the to-be-prolonged control. Finally, we provide the proof of our main theorem in 


\section{Section IV.}

\section{FLATNESS: DEFINITION AND KNOWN RESUlTS}

The fundamental property of flat systems is that all their solutions may be parametrized by a finite number of functions and their time-derivatives. Fix an integer $l \geq-1$ and denote $U^{l}=U \times \mathbb{R}^{m l}$ and $\bar{u}^{l}=\left(u, \dot{u}, \ldots, u^{(l)}\right)$. For $l=-1$, the set $U^{-1}$ is empty and $\bar{u}^{-1}$ in an empty sequence.

Definition 1: The system $\Xi: \dot{x}=F(x, u)$ is flat at $\left(x_{0}, \bar{u}_{0}^{l}\right) \in X \times U^{l}$, for $l \geq-1$, if there exists a neighborhood $\mathcal{O}^{l}$ of $\left(x_{0}, \bar{u}_{0}^{l}\right)$ and $m$ smooth functions $\varphi_{i}=\varphi_{i}\left(x, u, \dot{u}, \ldots, u^{(l)}\right), 1 \leq i \leq m$, defined in $\mathcal{O}^{l}$, having the following property: there exist an integer $s$ and smooth functions $\gamma_{i}, 1 \leq i \leq n$, and $\delta_{j}, 1 \leq j \leq m$, such that

$$
x_{i}=\gamma_{i}\left(\varphi, \dot{\varphi}, \ldots, \varphi^{(s-1)}\right) \text { and } u_{j}=\delta_{j}\left(\varphi, \dot{\varphi}, \ldots, \varphi^{(s)}\right)
$$

for any $C^{l+s}$-control $u(t)$ and corresponding trajectory $x(t)$ that satisfy $\left(x(t), u(t), \ldots, u^{(l)}(t)\right) \in \mathcal{O}^{l}$, where $\varphi=\left(\varphi_{1}, \ldots, \varphi_{m}\right)$ and is called a flat output.

Whenever necessary to specify the number of derivatives of $u$ on which the components of the flat outputs $\varphi$ depend, we say that the system $\Xi$ is $\left(x, u, \ldots, u^{(r)}\right)$ flat if $\varphi_{i}=\varphi_{i}\left(x, u, \ldots, u^{(r)}\right)$, for $1 \leq i \leq m$, and at least one of them depends explicitly on $u^{(r)}$. In the particular case $\varphi_{i}=\varphi_{i}(x)$, we say that the system is $x$-flat.

In general, $r$ is not bigger than the integer $l$ needed to define the neighborhood $\mathcal{O}^{l}$. In our study, $r$ will be proved to be -1 , i.e., the flat outputs depend on $x$ only, and $l$ is -1 or 0 .

The minimal number of derivatives of components of a flat output, needed to express $x$ and $u$, will be called the differential weight of that flat output and is formalized as follows. By definition, for any flat output $\varphi$ of $\Xi$ there exist integers $s_{1}, \ldots, s_{m}$ such that

$$
\begin{aligned}
x & =\gamma\left(\varphi_{1}, \dot{\varphi}_{1}, \ldots, \varphi_{1}^{\left(s_{1}\right)}, \ldots, \varphi_{m}, \dot{\varphi}_{m}, \ldots, \varphi_{m}^{\left(s_{m}\right)}\right) \\
u & =\delta\left(\varphi_{1}, \dot{\varphi}_{1}, \ldots, \varphi_{1}^{\left(s_{1}\right)}, \ldots, \varphi_{m}, \dot{\varphi}_{m}, \ldots, \varphi_{m}^{\left(s_{m}\right)}\right) .
\end{aligned}
$$

Moreover, we can choose $\left(s_{1}, \ldots, s_{m}\right), \gamma$ and $\delta$ such that (see [23]) if for any other $m$-tuple $\left(\tilde{s}_{1}, \ldots, \tilde{s}_{m}\right)$ and functions $\tilde{\gamma}$ and $\tilde{\delta}$, we have

$$
\begin{aligned}
x & =\tilde{\gamma}\left(\varphi_{1}, \dot{\varphi}_{1}, \ldots, \varphi_{1}^{\left(\tilde{s}_{1}\right)}, \ldots, \varphi_{m}, \dot{\varphi}_{m}, \ldots, \varphi_{m}^{\left(\tilde{s}_{m}\right)}\right) \\
u & =\tilde{\delta}\left(\varphi_{1}, \dot{\varphi}_{1}, \ldots, \varphi_{1}^{\left(\tilde{s}_{1}\right)}, \ldots, \varphi_{m}, \dot{\varphi}_{m}, \ldots, \varphi_{m}^{\left(\tilde{s}_{m}\right)}\right),
\end{aligned}
$$

then $s_{i} \leq \tilde{s}_{i}$, for $1 \leq i \leq m$. We will call $\sum_{i=1}^{m}\left(s_{i}+1\right)=$ $m+\sum_{i=1}^{m} s_{i}$ the differential weight of $\varphi$. A flat output of $\Xi$ is called minimal if its differential weight is the lowest among all flat outputs of $\Xi$. We define the differential weight of a flat system to be equal to the differential weight of a minimal flat output.

Consider a control-affine system

$$
\Sigma: \dot{x}=f(x)+\sum_{i=1}^{m} u_{i} g_{i}(x),
$$

where $f$ and $g_{1}, \cdots, g_{m}$ are smooth vector fields on $X$. The system $\Sigma$ is linearizable by static feedback if it is equivalent via a diffeomorphism $z=\phi(x)$ and an invertible static feedback transformation, $u=\alpha(x)+$ $\beta(x) v$, to a linear controllable system $\Lambda: \dot{z}=A z+B v$.

The problem of static feedback linearization was solved by Brockett [1] (for a smaller class of transformations) and then by Jakubczyk and Respondek [12] and, independently, by Hunt and Su [9], who gave geometric necessary and sufficient conditions. The following theorem recalls their result and, furthermore, gives an equivalent way of describing static feedback linearizable systems from the point of view of differential weight.

Define inductively the sequence of distributions $\mathcal{D}^{i+1}=\mathcal{D}^{i}+\left[f, \mathcal{D}^{i}\right]$, where $\mathcal{D}^{0}$ is given by $\mathcal{D}^{0}=$ $\operatorname{span}\left\{g_{1}, \cdots, g_{m}\right\}$ and $\left[f, \mathcal{D}^{i}\right]=\left\{[f, g]: g \in \mathcal{D}^{i}\right\}$.

Theorem 1: The following conditions are equivalent:

(FL1) $\Sigma$ is locally static feedback linearizable, around $x_{0} \in X$

(FL2) $\Sigma$ is locally static feedback equivalent, around $x_{0} \in X$, to the Brunovský canonical form

$$
(B r):\left\{\begin{array}{l}
\dot{z}_{i}^{j}=z_{i}^{j+1} \\
\dot{z}_{i}^{\rho_{i}}=v_{i}
\end{array}\right.
$$

where $1 \leq i \leq m, 1 \leq j \leq \rho_{i}-1$, and $\sum_{i=1}^{m} \rho_{i}=n$;

(FL3) For any $q \geq 0$, the distributions $\mathcal{D}^{q}$ are of constant rank, around $x_{0} \in X$, involutive and $\mathcal{D}^{n-1}=T X$

(FL4) $\Sigma$ is flat at $x_{0} \in X$, of differential weight $n+m$.

The geometry of static feedback linearizable systems is given by the following sequence of nested involutive distributions:

$$
\mathcal{D}^{0} \subset \mathcal{D}^{1} \subset \cdots \subset \mathcal{D}^{n-1}=T X .
$$

It is well known that a feedback linearizable system is static feedback equivalent to the Brunovský canonical form, see [2], and is clearly flat with $\varphi=$ $\left(\varphi_{1}, \cdots, \varphi_{m}\right)=\left(z_{1}^{1}, \cdots, z_{m}^{1}\right)$ being a minimal flat output (of differential weight $n+m$ ). Therefore, for static feedback linearizable systems, the representation of all states and controls uses the minimal possible, which is $n+m$, number of time-derivatives of $\varphi_{i}$ and an equivalent way of describing them is that they are flat systems of differential weight $n+m$.

In general, a flat system is not linearizable by static feedback, with the exception of the single-input case. Any single input-system is flat if and only if it is static feedback linearizable, see [4], [21], and thus of differential weight $n+1$. Flat systems can be seen as a generalization of static feedback linearizable systems. Namely they are linearizable via dynamic, invertible and endogenous feedback, see [6], [5], [13], [22]. In [16], [17], the authors described the simplest flat systems that are not static feedback linearizable: control-affine 
systems that become static feedback linearizable after a one-fold prolongation (which is the simplest dynamic feedback) thus of differential weight $n+m+1$. In this paper we will characterize systems (with two controls, i.e. $m=2$ ) that become static feedback linearizable after a two-fold prolongation, that is of differential weight $n+4$.

\section{MAIN RESULTS}

Throughout, we will consider two-input controlaffine systems of the form

$$
\Sigma: \dot{x}=f(x)+u_{1} g_{1}(x)+u_{2} g_{2}(x),
$$

where $x \in X, u=\left(u_{1}, u_{2}\right)^{t} \in \mathbb{R}^{2}$ and $f, g_{1}$, and $g_{2}$ are $\mathcal{C}^{\infty}$-smooth vector fields on $X$. We make the following assumption:

Assumption 1: From now on, unless stated otherwise, we assume that all ranks are constant in a neighborhood of a given $x_{0} \in X$. All results presented here are thus valid on an open and dense subset of either $X$ or $X \times U$, where all ranks are constant, and hold locally, around any point of that set.

Throughout, we deal only with systems that are not static feedback linearizable. Therefore one of distributions $\mathcal{D}^{i}$ fails to satisfy the condition (FL3) of Theorem 1 . Flat systems are always accessible so $\mathcal{D}^{n-1}=T X$ holds and all distributions $\mathcal{D}^{i}$ are supposed of constant rank according to Assumption 1. So there exists an integer $k$ such that $\mathcal{D}^{k}$ is not involutive. Suppose that $k$ is the smallest integer satisfying that property.

Flat systems of differential weight $n+4$ form a particular class of dynamic feedback linearizable systems, namely, they become static feedback linearizable after two-fold prolongations of a suitably chosen control. More precisely, we have the following result:

Proposition 1: Consider the system $\Sigma$, given by (3). The following conditions are equivalent:

(i) $\Sigma$ is flat at $\left(x_{0}, u_{0}, \dot{u}_{0}, \ldots, u_{0}^{(l)}\right)$, of differential weight $n+4$, for a certain $l \geq-1$;

(ii) $\Sigma$ is $x$-flat at either $x_{0}$ or $\left(x_{0}, u_{0}\right)$, of differential weight $n+4$;

(iii) There exists, around $x_{0}$, an invertible static feedback transformation $u=\alpha(x)+\beta(x) \tilde{u}$, bringing the system $\Sigma$ into the form $\tilde{\Sigma}: \dot{x}=\tilde{f}(x)+$ $\tilde{u}_{1} \tilde{g}_{1}(x)+\tilde{u}_{2} \tilde{g}_{2}(x)$, such that the prolongation

$$
\tilde{\Sigma}^{(2,0)}:\left\{\begin{array}{l}
\dot{x}=\tilde{f}(x)+y_{1} \tilde{g}_{1}(x)+v_{2} \tilde{g}_{2}(x) \\
\dot{y}_{1}=y_{2} \\
\dot{y}_{2}=v_{1}
\end{array}\right.
$$

is locally static feedback linearizable at $\left(x_{0}, y_{0}\right)$, with $y_{1}=\tilde{u}_{1}, v_{2}=\tilde{u}_{2}, \tilde{f}=f+\alpha g$ and $\tilde{g}=g \beta$, where $g=\left(g_{1}, g_{2}\right)$ and $\tilde{g}=\left(\tilde{g}_{1}, \tilde{g}_{2}\right)$.

A system $\Sigma$ satisfying (iii) will be called dynamically linearizable via an invertible two-fold prolongation. Notice that $\tilde{\Sigma}^{(2,0)}$ is, indeed, obtained by applying an invertible static feedback $u=\alpha+\beta \tilde{u}$ and then prolonging the first control $\tilde{u}_{1}$ twice as $v_{1}=\ddot{\tilde{u}}_{1}$ and not prolonging $\tilde{u}_{2}$ (which explains the notation). The above result asserts that, as for flatness of differential weight $n+3$ (see [16]), for systems of differential weight $n+4$, flatness and $x$-flatness coincide and that, moreover, they are equivalent to linearizability via twofold prolongations of a suitably chosen control.

Before giving our main results, we state the following proposition needed in the proofs, but also having an independent interest.

Proposition 2: Consider the system $\Sigma$, given by (3), dynamically linearizable via invertible two-fold prolongation and let $\mathcal{D}^{k}$ be its first noninvolutive distribution. Then the distribution $\mathcal{D}^{k}$ is feedback invariant and $\operatorname{cork}\left(\mathcal{D}^{k} \subset \overline{\mathcal{D}}^{k}\right) \leq 2$.

According to the above proposition, at most two directions of $\overline{\mathcal{D}}^{k}$ stick out of $\mathcal{D}^{k}$. In this paper, we will study only the case when the noninvolutivity of $\mathcal{D}^{k}$ is maximal, i.e., when cork $\left(\mathcal{D}^{k} \subset \overline{\mathcal{D}}^{k}\right)=2$. The particular case cork $\left(\mathcal{D}^{k} \subset \overline{\mathcal{D}}^{k}\right)=1$ needs a separate analysis, it reminds the results for flatness of differential weight $n+3$, but it has a slightly different geometry. In order to respect the length of conference paper, we do not discuss it here. Moreover, we suppose $\overline{\mathcal{D}}^{k} \neq T X$. The particular cases, cork $\left(\mathcal{D}^{k} \subset \overline{\mathcal{D}}^{k}\right)=1$ and $\overline{\mathcal{D}}^{k}=T X$ will be presented in a future paper. Thus, from now on, we make the following assumption

Assumption 2: We assume cork $\left(\mathcal{D}^{k} \subset \overline{\mathcal{D}}^{k}\right)=2$ and $\overline{\mathcal{D}}^{k} \neq \mathrm{TX}$.

Remark 1: If cork $\left(\mathcal{D}^{k} \subset \overline{\mathcal{D}}^{k}\right)=2$, then it can be shown that we necessarily have $r k \mathcal{D}^{k}=2 k+2$ (see Proposition 4, in Section IV).

Our main result describing flat systems of differential weight $n+4$ is given by the three following theorems corresponding to the first noninvolutive distribution $\mathcal{D}^{k}$ being either $\mathcal{D}^{0}$, i.e., $k=0$ (Theorem 3 ) or $\mathcal{D}^{1}$, i.e., $k=1$ (Theorem 4 ) or $\mathcal{D}^{k}$, for $k \geq 2$ (Theorem 2). For all theorems, we assume that Assumptions 1 and 2 hold.

Theorem 2: Assume that $k \geq 2$ and consider the twoinput control system $\Sigma$, given by (3). The system $\Sigma$ is flat at $x_{0}$, of differential weight $n+4$, if and only if the following conditions hold:

(A1) $\operatorname{rk}\left(\overline{\mathcal{D}}^{k}+\left[f, \mathcal{D}^{k}\right]\right)=2 k+5$, implying the existence of a non-zero vector field $g_{2 c} \in \mathcal{D}^{0}$ such that $a d_{f}^{k+1} g_{2 c} \in \overline{\mathcal{D}}^{k}$

(A2) $a d_{f}^{k+2} g_{2 c} \in \overline{\mathcal{D}}^{k}+\mathcal{D}^{k+1}$;

(A3) The distribution $\mathcal{L}^{k}=\operatorname{span}\left\{a d_{f_{c}}^{q} g_{1 c}, a d_{f_{c}}^{r} g_{2 c}, 0 \leq\right.$ $q \leq k-2,0 \leq r \leq k\}$ is involutive, where $g_{2 c}$ is defined by condition (A1), $g_{1 c} \in \mathcal{D}^{0}$ is independent of $g_{2 c}$ and such that $\left[g_{1 c}, g_{2 c}\right]=$ 0 mod span $\left\{g_{2 c}\right\}$ and $f_{c}$ satisfies $a d_{f_{c}}^{k+2} g_{2 c} \in \overline{\mathcal{D}}^{k}$;

(A4) The distributions $\mathcal{L}^{i+1}=\mathcal{L}^{i}+\left[f_{c}, \mathcal{L}^{i}\right]$, for $i \geq k$, are involutive; 
(A5) There exists $\rho$ such that $\mathcal{L}^{\rho}=T X$.

The geometry of the systems described by the previous theorem can be summarized by the following sequence of inclusions:

$$
\begin{aligned}
& \begin{array}{r}
\mathcal{D}^{0} \underset{2}{\subset} \cdots \underset{2}{\subset} \mathcal{D}^{k-1} \underset{2}{\subset} \mathcal{D}^{k} \underset{2}{\subset} \overline{\mathcal{D}}^{k} \\
2 \cup 1 \cup
\end{array} \\
& \mathcal{L}^{k} \underset{2}{\subset} \mathcal{L}^{k+1} \underset{2}{\subset} \mathcal{L}^{k+2} \cdots \subset_{2} \mathcal{L}^{\mu} \subset_{1} \mathcal{L}^{\mu+1} \cdots \underset{1}{\subset} \mathcal{L}^{\rho}=T X
\end{aligned}
$$

where all distributions, except $\mathcal{D}^{k}$, are involutive and the integers beneath the inclusion symbol " $C$ " indicate coranks. According to condition $(A 1)$, if we take the brackets of $\mathcal{D}^{k}$ with $f$, we gain only one new direction with respect to $\overline{\mathcal{D}}^{k}$ implying the existence of a distinguished vector field $g_{2 c}$ in $\mathcal{D}^{0}$ that allows us to define another vector field $g_{1 c} \in \mathcal{D}^{0}$ and a distinguished drift $f_{c}$ that is used to define the involutive subdistribution $\mathcal{L}^{k}$ of corank two in $\mathcal{D}^{k}$ (which is essential for flatness of differential weight $n+4)$. While, the computation of $g_{2 c}$ involves derivations and algebraic operations only, calculating $g_{1 c}$ and $f_{c}$ requires solving two partial differential equations (which always admit solutions), defined and discussed in Section III-A. Contrary to flatness of differential weight $n+3$, for which only the definition of a control vector field $g_{2 c}$ is needed (see [16]), for flatness of differential weight $n+4$, it is necessary to distinguish also $g_{1 c}$ and $f_{c}$. Indeed, it can be shown that not all control vector fields $g_{1}$ and not all drifts $f$ lead to a static feedback linearizable prolongation $\Sigma^{(2,0)}$. This can also be observed when proving the above theorem, see Section IV.

The geometry of flat systems of differential weight $n+4$ recalls very much that of flat systems of differential weight $n+3$, see [16]. Similarly to flat systems of differential weight $n+3$, for which the the main structural condition is the existence of an involutive subdistribution $\mathcal{H}^{k}$ of corank one in $\mathcal{D}^{k}$, see [16], the most important structural property of flat systems of differential weight $n+4$ is the existence of an involutive subdistribution $\mathcal{L}^{k}$ of corank two in $\mathcal{D}^{k}$. Indeed, similarly to $\mathcal{H}^{k}$, the distribution $\mathcal{L}^{k}$ is essential: it takes the role of the noninvolutive distribution $\mathcal{D}^{k}$ and, moreover, its successive brackets with the distinguished drift $f_{c}$ are again involutive (replacing the distributions $\mathcal{D}^{k+i}$ ). Contrary to $\mathcal{H}^{k}$, the definition of $\mathcal{L}^{k}$ is more involved since, in order to construct $\mathcal{L}^{k}$, we have to compute the vector fields $g_{1 c}$ and $f_{c}$ and, therefore, to solve some PDE's (that always admit solutions). The involutive subdistribution $\mathcal{L}^{k}$ is invariant under feedback transformations consistent with the definitions of $g_{1 c}, g_{2 c}$ and $f_{c}$. Notice that the sequence of inclusions between the distributions $\mathcal{L}^{i}$ and $\mathcal{D}^{i}$ is no longer as simple as between $\mathcal{H}^{i}$ and $\mathcal{D}^{i}$. Indeed, in the latter case, we simply have $\mathcal{D}^{k-1} \underset{1}{\subset} \mathcal{H}^{k} \subset_{1} \mathcal{D}^{k} \subset \overline{\mathcal{D}}^{k}=\mathcal{H}^{k+1}$, while the counterpart of that sequence, in the case of $\mathcal{L}^{i}$ and $\mathcal{D}^{i}$, can be written as

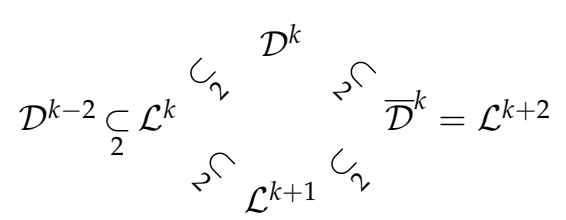

It is easy to check that $\overline{\mathcal{D}}^{k}=\mathcal{L}^{k+2}$. Indeed, by definition, $\mathcal{L}^{k+2}=\mathcal{D}^{k}+\operatorname{span}\left\{a d_{f_{c}}^{k+1} g_{2 c}, a d_{f_{c}}^{k+2} g_{2 c}\right\}$ and its involutivity is given by $(A 4)$. Moreover, $\mathrm{rk} \mathcal{L}^{k+2}=2 k+$ 4 , otherwise $\mathcal{D}^{k}$ would be involutive or cork $\left(\mathcal{D}^{k} \subset \overline{\mathcal{D}}^{k}\right)$ would be one. It follows that $\overline{\mathcal{D}}^{k}=\mathcal{L}^{k+2}$. Thus the directions completing $\mathcal{D}^{k}$ to $\overline{\mathcal{D}}^{k}$ are, modulo $\mathcal{D}^{k}$, in the plane spanned by $a d_{f_{c}}^{k+1} g_{2 c}$ and $a d_{f_{c}}^{k+2} g_{2 c}$.

If $k=0$ or $k=1$, i.e., the first noninvolutive distribution is $\mathcal{D}^{0}$ or $\mathcal{D}^{1}$, then a similar result holds, but in the chain of involutive subdistributions $\mathcal{L}^{1} \subset \mathcal{L}^{2} \subset$ $\cdots$ (playing the role of $\mathcal{L}^{k} \subset \mathcal{L}^{k+1} \subset \mathcal{L}^{k+2} \subset \cdots$ ), the distributions $\mathcal{L}^{1}$ and $\mathcal{L}^{2}$ are not defined as $\mathcal{L}^{k+1}$ and $\mathcal{L}^{k+2}$ (see condition $(A 4)^{\prime}$ of Theorem 3, resp. the statement of Theorem 4). Moreover, flat systems with $k=0$ or $k=1$ exhibit singularities in the control space (created by a two-fold prolongation of the tobe-prolonged control and excluded by condition $(R C)$ ) which are defined by

$U_{\text {sing }}(x)=\left\{u \in \mathbb{R}^{2}:\left(g_{1 c} \wedge g_{2 c} \wedge a d_{f_{c}+g_{c} u} g_{2 c} \wedge\right.\right.$
$\left.\left.a d_{f_{c}+g_{c} u}^{2} g_{2 c}\right)(x)=0\right\}$, with $f_{c}+g_{c} u=f_{c}+g_{1 c} u_{1}+g_{2 c} u_{2}$, where the vector fields $f_{c}, g_{1 c}$ and $g_{2 c}$ are defined in the statements of Theorems 3 and 4 .

Theorem 3: Assume that $k=0$ and consider the twoinput control system $\Sigma$, given by (3). The system $\Sigma$ is flat at $\left(x_{0}, u_{0}\right)$, of differential weight $n+4$, if and only if the following conditions hold:

(A1)' The ranks of the distributions $\mathcal{G}^{0}=\mathcal{D}^{0}, \mathcal{G}^{1}=$ $\mathcal{G}^{0}+\left[\mathcal{G}^{0}, \mathcal{G}^{0}\right]$, and $\mathcal{G}^{2}=\mathcal{G}^{1}+\left[\mathcal{G}^{0}, \mathcal{G}^{1}\right]$ are, respectively two, three, and four implying the existence of a characteristic vector field $g_{2 c} \in \mathcal{D}^{0}$ for the distribution $\mathcal{G}^{1}$, i.e., $\left[g_{2 c}, \mathcal{G}^{1}\right] \in \mathcal{G}^{1}$;

(A2)' $a d_{f} g_{2 c} \in \mathcal{G}^{1}$;

(A3)' $a d_{f}\left[g_{1}, g_{2 c}\right], a d_{f}^{2} g_{2 c} \in \overline{\mathcal{D}}^{0}+\left[f, \mathcal{D}^{0}\right]$;

(A4)' The distributions $\mathcal{L}^{i}$, for $i \geq 2$, are involutive, where $\mathcal{L}^{2}=\overline{\mathcal{D}}^{0}$ and $\mathcal{L}^{i+1}=\mathcal{L}^{i}+\left[f, \mathcal{L}^{i}\right]$, for $i \geq 2$;

(A5)' There exists $\rho$ such that $\mathcal{L}^{\rho}=T X$;

(RC) $u_{0} \notin U_{\text {sing }}\left(x_{0}\right)$, where $g_{2 c}$ is defined by condition (A1), $g_{1 c} \in \mathcal{D}^{0}$ is independent of $g_{2 c}$ and such that span $\left\{g_{2 c},\left[g_{1 c}, g_{2 c}\right]\right\}$ is involutive and $f_{c}$ satisfies $a d_{f_{c}} g_{2 c} \in \operatorname{span}\left\{g_{2 c},\left[g_{1 c}, g_{2 c}\right]\right\}$.

Notice that the involutive distribution span $\left\{g_{2 c},\left[g_{1 c}, g_{2 c}\right]\right.$ plays the role of $\mathcal{L}^{k+1}$ for the case $k \geq 2$. Since the distribution $\mathcal{D}^{0}$ is supposed noninvolutive, the vector field $g_{1 c}$ cannot be defined in the same way as for the case $k \geq 2$. Consequently, the definition of $f_{c}$ changes as well. 
Theorem 4: Assume $k=1$ and consider the twoinput control system $\Sigma$, given by (3). The system $\Sigma$ is flat at $\left(x_{0}, u_{0}\right)$, of differential weight $n+4$, if and only if conditions $(A 1)-(A 5)$ of Theorem 2 hold for $k=1$, with $\mathcal{L}^{1}=\operatorname{span}\left\{g_{2 c}, a d_{f_{c}} g_{2 c}\right\}, \mathcal{L}^{2}=$ span $\left\{g_{1 c}, g_{2 c}, a d_{f_{c}} g_{2 c}, a d_{f_{c}}^{2} g_{2 c},\left[g_{1 c}, a d_{f_{c}} g_{2 c}\right]\right\}$, and $\mathcal{L}^{i}$, for $i \geq 3$, defined as in $(A 4)$, and $u_{0} \notin U_{\text {sing }}\left(x_{0}\right)$.

The conditions of our theorems enable us to define the control to be prolonged in order to obtain a locally static feedback linearizable prolongation $\tilde{\Sigma}^{(2,0)}$. We explain in the next subsection, the construction of that control.

\section{A. To-be-prolonged control}

In this section, we will define the control $u_{1 p}$ to be prolonged (preintegrated) in order to dynamically linearize the system. We will study only the cases $k \geq 2$ and $k=1$. If the first noninvolutive distribution is $\overline{\mathcal{D}}^{0}$, then a similar analysis can be done.

Let us first show that under the condition $(A 1)$ of Theorem 2 , there exists a non-zero vector field $g_{2 c} \in \mathcal{D}^{0}$ such that $a d_{f}^{k+1} g_{2 c} \in \overline{\mathcal{D}}^{k}$. We have $\operatorname{rk} \overline{\mathcal{D}}^{k}=2 k+4$ and, accoording to $(A 1), \operatorname{rk}\left(\overline{\mathcal{D}}^{k}+\left[f, \mathcal{D}^{k}\right]\right)=2 k+5$, thus we can always assume (permute $g_{1}$ and $g_{2}$, if necessary) that $a d_{f}^{k+1} g_{1} \notin \overline{\mathcal{D}}^{k}$. Hence there exists a smooth function $v$, defined in a neighborhood of $x_{0}$, such that $a d_{f}^{k+1} g_{2}=\operatorname{vad}_{f}^{k+1} g_{1} \bmod \overline{\mathcal{D}}^{k}$. The vector field $g_{2 c}=g_{2}-v g_{1}$ is obviously nonzero (since $g_{1}$ and $g_{2}$ are independent everywhere on $X$ ) and satisfies $a d_{f}^{k+1} g_{2 c} \in \overline{\mathcal{D}}^{k}$ and it is clear that any vector field $\tilde{g}_{2 c}=\beta_{1} g_{1}+\beta_{2} g_{2}$, collinear with $g_{2}-v g_{1}$ satisfies $a d_{f}^{k+1} \tilde{g}_{2 c} \in \overline{\mathcal{D}}^{k}$. It follows that $g_{2 c}$ is not unique and all vector fields $g_{2 c}$ form a subdistribution of $\mathcal{D}^{0}=$ span $\left\{g_{1}, g_{2}\right\}$ of rank one.

We explain next how to find the vector field $g_{1 c}=$ $\beta g_{1}+\gamma g_{2 c} \in \mathcal{D}^{0}$, independent of $g_{2 c}$, and such that $\left[g_{1 c}, g_{2 c}\right]=0 \bmod \operatorname{span}\left\{g_{2 c}\right\}$. Since $k \geq 1$, the distribution $\mathcal{D}^{0}$ is involutive and there exists a smooth function $\lambda$ such that $\left[g_{1}, g_{2 c}\right]=\lambda g_{1} \bmod \operatorname{span}\left\{g_{2 c}\right\}$. Then the function $\beta$ has to satisfy $L_{g_{2 c}} \beta-\beta \lambda=0$ (which always admits solutions, so $\beta$ always exists) and $\gamma$ can be any. It is immediate that any vector field $\tilde{g}_{1 c}=\tilde{\beta} g_{1 c}+\tilde{\gamma} g_{2 c}$, where the function $\tilde{\beta}$ is non zero and satisfies that $L_{g_{2 c}} \tilde{\beta}=0$ and $\tilde{\gamma}$ is any, verifies $\left[\tilde{g}_{1 c}, g_{2 c}\right]=0 \bmod \operatorname{span}\left\{g_{2 c}\right\}$.

Now, we are looking for a couple of functions $\left(\alpha_{1}, \alpha_{2}\right)$ such that $f_{c}=f+\alpha_{1} g_{1 c}+\alpha_{2} g_{2 c}$ satisfies $a d_{f_{c}}^{k+2} g_{2 c} \in \overline{\mathcal{D}}^{k}$. Using Proposition 5 (see Section IV), it can be proven that $a d_{f_{c}}^{k+2} g_{2 c}=a d_{f}^{k+2} g_{2 c}-$ $\left(L_{g_{2 c}} \alpha_{1}\right) a d_{f}^{k+1} g_{1 c} \bmod \overline{\mathcal{D}}^{k}$. According to item (A2) of Theorem 2, there exists a smooth function $\delta$ such that $a d_{f}^{k+2} g_{2 c}=\delta a d_{f}^{k+1} g_{1 c} \bmod \overline{\mathcal{D}}^{k}$. We deduce that the function $\alpha_{1}$ has to satisfy $L_{g_{2 c}} \alpha_{1}=\delta$ (which always admits solutions, so $\alpha_{1}$ always exists) and $\alpha_{2}$ can be any. Notice that any vector field $\tilde{f}_{c}=f_{c}+\tilde{\alpha}_{1} g_{1 c}+\tilde{\alpha}_{2} g_{2 c}$, where $\tilde{\alpha}_{1}$ is such that $L_{g_{2 c}} \tilde{\alpha}_{1}=0$ and $\tilde{\alpha}_{2}$ is any, verifies $a d_{\tilde{f}_{c}}^{k+2} g_{2 c} \in \overline{\mathcal{D}}^{k}$.

We define the to-be-prolonged control by the relation:

$$
\left(\begin{array}{l}
u_{1} \\
u_{2}
\end{array}\right)=\left(\begin{array}{c}
\alpha_{1}(\beta-\gamma v)-v \alpha_{2} \\
\alpha_{1} v+\alpha_{2}
\end{array}\right)+\left(\begin{array}{cc}
\beta-\gamma v & -v \\
\gamma & 1
\end{array}\right)\left(\begin{array}{l}
u_{1 p} \\
u_{2 p}
\end{array}\right) .
$$

Consider the system

$$
\Sigma_{c}: \dot{x}=f_{c}+u_{1 p} g_{1 c}+u_{2 p} g_{2 c}
$$

which is static feedback equivalent to the original system $\Sigma$, and its prolongation

$$
\Sigma_{c}^{(2,0)}:\left\{\begin{array}{l}
\dot{x}=f_{c}(x)+u_{1 p}^{0} g_{1 c}(x)+v_{2} g_{2 c}(x) \\
\dot{u}_{1 p}^{0}=u_{1 p}^{1} \\
\dot{u}_{1 p}^{1}=v_{1}
\end{array}\right.
$$

controlled by $\left(v_{1}, v_{2}\right)=\left(\ddot{u}_{1 p}, u_{2 p}\right)$ and evolving on the prolonged state space $X_{p}=X \times \mathbb{R}^{2}$, where $\left(x, u_{1 p}^{0}, u_{1 p}^{1}\right) \in X \times \mathbb{R}^{2}$. We can now restate all our theorems using just one statement for the prolonged system.

Theorem 5: The control system $\Sigma$ is flat at $\left(x_{0}, u_{0}\right)$ of differential weight $n+4$ if and only if the prolonged system $\Sigma_{c}^{(2,0)}$ is locally static feedback linearizable around $\left(x_{0}, u_{p 0}^{0}, u_{p 0}^{1}\right)$, i.e., it satisfies the equivalent conditions of Theorem 1.

\section{B. Normal form}

The following proposition gives a normal form for the class of two-input flat systems of differential weight $n+4$. The integers $\rho_{i}$ that show up in the normal form are related to $\rho$ and $\mu$ defined via the nested sequence of distributions $\mathcal{L}^{i}$. Let $\mu$ be the smallest integer such that corank $\left(\mathcal{L}^{\mu} \subset \mathcal{L}^{\mu+1}\right)$ is one and $\rho$ is the smallest integer such that $\mathcal{L}^{\rho}=T X$. It follows that $\rho+\mu=n$. Define the pair of indices $\left(\rho_{1}, \rho_{2}\right)$ by $\rho=\max \left(\rho_{1}, \rho_{2}+\right.$ 2 ) and $\mu=\min \left(\rho_{1}, \rho_{2}+2\right)$. We have $\rho_{1}+\rho_{2}+2=$ $n$, implying $\rho_{i} \geq k+1$, and since we have supposed $\overline{\mathcal{D}}^{k} \neq T X$, at least one of them is greater than $k+2$. For $i=1,2$, denote $\bar{z}_{i}^{j}=\left(z_{i}^{1}, \cdots, z_{i}^{j}\right)$.

Proposition 3: The system $\Sigma$ is flat at $x_{0}\left(\right.$ at $\left(x_{0}, u_{0}\right)$, such that $u_{0}=\left(u_{10}, u_{20}\right) \notin U_{\text {sing }}\left(x_{0}\right)$, if $k=0$ or 1$)$ of differential weight $n+4$ if and only if it is locally, around $x_{0}$, static feedback equivalent to the following normal form in a neighborhood $Z$ of $z_{0} \in \mathbb{R}^{n}$ :

$(N F)\left\{\begin{array}{cll}\dot{z}_{1}^{1}=z_{1}^{2} & \dot{z}_{2}^{1}=z_{2}^{2} \\ \vdots & & \vdots \\ \dot{z}_{1}^{\rho_{1}-1}=z_{1}^{\rho_{1}} & \dot{z}_{2}^{\rho_{2}-1}=z_{2}^{\rho_{2}} \\ \dot{z}_{1}^{\rho_{1}}=\tilde{u}_{1} & \dot{z}_{2}^{\rho_{2}}=a\left(\bar{z}_{1}^{\rho_{1}}, \bar{z}_{2}^{\rho_{2}+1}\right)+b\left(\bar{z}_{1}^{\rho_{1}}, \bar{z}_{2}^{\rho_{2}+1}\right) \tilde{u}_{1} \\ & & \dot{z}_{2}^{\rho_{2}+1}=c(z)+d(z) \tilde{u}_{1} \\ & \dot{z}_{2}^{\rho_{2}+2}=\tilde{u}_{2}\end{array}\right.$

where 
- either $k=0$ and then $b=z_{2}^{\rho_{2}+1}, d=z_{2}^{\rho_{2}+2}, a$ and $c$ are any functions ( $a$ not depending on $z_{2}^{\rho_{2}+2}$ ) satisfying $\frac{\partial a}{\partial z_{2}^{\rho_{2}+1}}\left(z_{0}\right)+u_{10} \neq 0, \frac{\partial c}{\partial z_{2}^{\rho_{2}+2}}\left(z_{0}\right)+u_{10} \neq 0$;

- or $k=1$ and then $c=z_{2}^{\rho_{2}+2}, d=d\left(\bar{z}_{1}^{\rho_{1}}, \bar{z}_{2}^{\rho_{2}+1}\right)$ and either $a=z_{2}^{\rho_{2}+1}$ and $b$ is any function, not depending on $z_{2}^{\rho_{2}+2}$, and satisfying $u_{10} \frac{\partial b}{\partial z_{2}^{\rho_{2}+1}}\left(z_{0}\right)+1 \neq 0$ or $b=z_{2}^{\rho_{2}+1}$ and $a$ is any function, not depending on $z_{2}^{\rho_{2}+2^{2}}$, and satisfying $\frac{\partial a}{\partial z_{2}^{\rho_{2}+1}}\left(z_{0}\right)+u_{10} \neq 0$;

- or $k \geq 2$ and then $a=z_{2}^{\rho_{2}+1}, c=z_{2}^{\rho_{2}+2}$, $b=b\left(\bar{z}_{1}^{\rho_{1}-k+2}, \bar{z}_{2}^{\rho_{2}-k+2}\right), d=d\left(\bar{z}_{1}^{\rho_{1}-k+2}, \bar{z}_{2}^{\rho_{2}-k+2}\right)$ and $\frac{\partial b}{\partial z_{1}^{\rho_{1}-k+2}}+b \frac{\partial b}{\partial z_{2}^{\rho_{2}-k+2}}=0, \frac{\partial d}{\partial z_{1}^{\rho_{1}-k+2}}-\frac{\partial^{2}}{\partial z_{1}^{\rho_{1}-k+1}}-$ $b \frac{\partial b}{\partial z_{2}^{\rho_{2}-k+1}}+b \frac{\partial d}{\partial z_{2}^{\rho_{2}-k+2}}-d \frac{\partial b}{\partial z_{2}^{\rho_{2}-k+2}}=0$;

and with $a, b, c$ and $d$ such that the distribution $\mathcal{D}^{k}$ is noninvolutive and cork $\left(\mathcal{D}^{k} \subset \overline{\mathcal{D}}^{k}\right)=2$, for $k \geq 1$.

Moreover, the minimal $x$-flat outputs and the normal form (NF) are compatible: if $\left(\varphi_{1}, \varphi_{2}\right)$ is a minimal $x$ flat output at $x_{0}$, then there exists an invertible static feedback transformation bringing the system $\Sigma$ into (NF) with $\varphi_{1}=z_{1}^{1}$ and $\varphi_{2}=z_{2}^{1}$.

At first sight there are four arbitrary functions involved in the normal form, but, in fact, we can always normalize two of them. This is due to the fact that $\mathcal{D}^{k}$ is squeezed between two involutive distributions $\mathcal{L}^{k}$ and $\overline{\mathcal{D}}^{k}=\mathcal{L}^{k+2}$ and both inclusions are of corank two (see the sequence of inclusions summarizing the geometry of flat systems of differential weight $n+4$ ), so only two directions of $\overline{\mathcal{D}}^{k}$ stick out of $\mathcal{D}^{k}$. The normal form (NF) is valid around $z_{0} \in \mathbb{R}^{n}$, which may be zero or not. Therefore it can be used around any point (equilibrium or not). It is immediate to see that (NF) is flat with $\varphi=\left(z_{1}^{1}, z_{2}^{1}\right)$ being a minimal flat output and a simple computation shows that its differential weight is, indeed, $n+4$. It is clear that (NF) becomes locally static feedback linearizable after a twofold prolongation of $\tilde{u}_{1}$. The normal form applies to all cases $k \geq 2, k=1$ and $k=0$, and it is clear that in the cases $k=1$ and $k=0$ (and only in those cases!), the precompensator creates singularities in the control space (depending on the state). Indeed, the controls $\tilde{u}_{0}$ satisfying $\frac{\partial a}{\partial z_{2}^{\rho_{2}+1}}\left(z_{0}\right)+\tilde{u}_{10}=0$ or $\frac{\partial c}{\partial z_{2}^{\rho_{2}+2}}\left(z_{0}\right)+u_{10}=0$ (resp. $\frac{\partial a}{\partial z_{2}^{\rho_{2}+1}}\left(z_{0}\right)+\tilde{u}_{10} \frac{\partial b}{\partial z_{2}^{\rho_{2}+1}}\left(z_{0}\right)=0$, where either $a=$ $z_{2}^{\rho_{2}+1}$ or $b=z_{2}^{\rho_{2}+1}$ ) are singular for (NF), if $k=0$ (resp. if $k=1$ ). We do not give here explicit conditions for $a$, $b, c$ and $d$ such that the distribution $\mathcal{D}^{k}$ is noninvolutive and $\operatorname{cork}\left(\mathcal{D}^{k} \subset \overline{\mathcal{D}}^{k}\right)=2$ for the cases $k \geq 2$ and $k=1$. They can be found by a straightforward computation.

\section{PROOFS}

\section{A. Notations and useful results}

Consider a control system of the form $\Sigma: \dot{x}=$ $f(x)+u_{1} g_{1}(x)+u_{2} h_{2}(x)$. By $\Sigma^{(2,0)}$ we will denote the system $\Sigma$ with two-fold prolongation of the first control, that is,

$$
\Sigma^{(2,0)}:\left\{\begin{array}{l}
\dot{x}=f(x)+y_{1} g_{1}(x)++v_{2} h_{2}(x) \\
\dot{y}_{1}=y_{2} \\
\dot{y}_{2}=v_{1}
\end{array}\right.
$$

with $y_{1}=u_{1}$ and $v_{2}=u_{2}$. The first control is prolonged, but the second is not, thus the corresponding vector fields do not play the same role and hence we denote them by $g_{1}$ and $h_{2}$ instead of $g_{1}$ and $g_{2}$. Denote by $f_{p}, g_{1 p}$ and $g_{2 p}$, respectively, the drift and the control vector fields of the prolonged canonical system $\Sigma^{(2,0)}$, i.e., $f_{p}=\sum_{i=1}^{n}\left(f_{i}+y_{1} g_{1 i}\right) \frac{\partial}{\partial x_{i}}+y_{2} \frac{\partial}{\partial y_{1}}, g_{1 p}=\frac{\partial}{\partial y_{2}}$ and $g_{2 p}=\sum_{i=1}^{n} h_{2 i} \frac{\partial}{\partial x_{i}}$. To $\Sigma^{(2,0)}$, we associate the distributions $\mathcal{D}_{p}^{0}=\operatorname{span}\left\{g_{1 p}, g_{2 p}\right\}$ and $\mathcal{D}_{p}^{i+1}=\mathcal{D}_{p}^{i}+\left[f_{p}, \mathcal{D}_{p}^{i}\right]$, for $i \geq 0$ (the subindex $p$ referring to the prolonged system $\left.\Sigma^{(1,0)}\right)$.

We start by stating two propositions needed in the proofs of our main results, but also having an independent interest. Proposition 4 is a complete version of Proposition 2.

Proposition 4: Consider the system $\Sigma$, given by (3), dynamically linearizable via invertible two-fold prolongation and let $\mathcal{D}^{k}$ be its first noninvolutive distribution. Then the following conditions are satisfied

(i) $\mathcal{D}^{k}$ is feedback invariant;

(ii) If $k \geq 2$, then $\operatorname{cork}\left(\mathcal{D}^{k-2} \subset \mathcal{D}^{k-1}\right)=2$;

(iii) $\operatorname{cork}\left(\mathcal{D}^{k} \subset \overline{\mathcal{D}}^{k}\right) \leq 2$;

(iv) If $\operatorname{cork}\left(\mathcal{D}^{k} \subset \overline{\mathcal{D}^{k}}\right)=2$, then $\operatorname{rk} \mathcal{D}^{k}=2 k+2$.

Proposition 5: Consider the system $\Sigma$, given by (3), and let $\mathcal{D}^{k}$ be its first noninvolutive distribution. Assume $k \geq 2$ and that conditions $(A 1)-(A 3)$ of Theorem 2 are satisfied. Then the distribution $\mathcal{H}^{k-1}=$ $\mathcal{D}^{k-2}+\operatorname{span}\left\{a d_{f}^{k-1} g_{2 c}\right\}$ is involutive and its involutivity implies that of all istributions $\mathcal{H}^{i}=\mathcal{D}^{i-1}+$ span $\left\{a d_{f}^{i} g_{2 c}\right\}$, for $1 \leq i \leq k-2$. Moreover, all distributions $\mathcal{L}^{i}=\operatorname{span}\left\{a d_{f_{c}}^{q} g_{1 c}, a d_{f_{c}}^{r} g_{2 c}, 0 \leq q \leq i-2,0 \leq r \leq\right.$ $i\}$, for $1 \leq i \leq k-1$ (where $\mathcal{L}^{1}=\operatorname{span}\left\{g_{2 c}, a d_{f_{c}} g_{2 c}\right\}$ ), are involutive.

\section{B. Proof of Theorem 2}

Necessity. Consider the control system $\Sigma: \dot{x}=$ $f(x)+u_{1} g_{1}(x)+u_{2} g_{2}(x)$ and assume that it is flat of differential weight $n+4$. According to Proposition 1, there exists an invertible static feedback transformation $u=\alpha(x)+\beta(x) \tilde{u}$, bringing $\Sigma$ into the form $\tilde{\Sigma}: \dot{x}=$ $\tilde{f}(x)+\tilde{u}_{1} \tilde{g}_{1}(x)+\tilde{u}_{2} \tilde{h}_{2}(x)$, such that the prolongation

$$
\tilde{\Sigma}^{(2,0)}:\left\{\begin{array}{l}
\dot{x}=\tilde{f}(x)+y_{1} \tilde{g}_{1}(x)+v_{2} \tilde{h}_{2}(x) \\
\dot{y}_{1}=y_{2} \\
\dot{y}_{2}=v_{1}
\end{array}\right.
$$


with $y_{1}=\tilde{u}_{1}$ and $v_{2}=\tilde{u}_{2}$, is locally static feedback linearizable. For simplicity of notation, we will drop the tildes, but we will keep distinguishing $g_{1}$ from $h_{2}$ (which could also be denoted $g_{2}$ ) whose control is not preintegrated.

Since $\Sigma^{(2,0)}$ is locally static feedback linearizable, for any $i \geq 0$, the distributions $\mathcal{D}_{p}^{i}$ are involutive, of constant rank, and there exists an integer $\rho$ such that $\operatorname{rk} \mathcal{D}_{p}^{\rho}=n+1$. We have

$$
\begin{aligned}
& \mathcal{D}_{p}^{0}=\operatorname{span}\left\{\frac{\partial}{\partial y_{2}}, h_{2}\right\}, \\
& \mathcal{D}_{p}^{1}=\operatorname{span}\left\{\frac{\partial}{\partial y_{1}}, \frac{\partial}{\partial y_{2}}, h_{2}, a d_{f} h_{2}+y_{1}\left[g_{1}, h_{2}\right]\right\} .
\end{aligned}
$$

Since $k \geq 2$ and $\operatorname{rk} \mathcal{D}^{1}=4$, we deduce from the involutivity of $\mathcal{D}_{p}^{1}$, that we necessarily have $\left[g_{1}, h_{2}\right]=0 \bmod \operatorname{span}\left\{h_{2}\right\}$ and that $\mathcal{D}_{p}^{1}=$ $\operatorname{span}\left\{\frac{\partial}{\partial y_{1}}, \frac{\partial}{\partial y_{2}}, h_{2}, a d_{f} h_{2}\right\}$. The distribution

$\mathcal{D}_{p}^{2}=\operatorname{span}\left\{\frac{\partial}{\partial y_{1}}, \frac{\partial}{\partial y_{2}}, g_{1}, h_{2}, a d_{f} h_{2}, a d_{f}^{2} h_{2}+y_{1}\left[g_{1}, a d_{f} h_{2}\right]\right\}$

is involutive, thus we actually have $\mathcal{D}_{p}^{2}=$ $\operatorname{span}\left\{\frac{\partial}{\partial y_{1}}, \frac{\partial}{\partial y_{2}}, g_{1}, h_{2}, a d_{f} h_{2}, a d_{f}^{2} h_{2},\left[g_{1}, a d_{f} h_{2}\right]\right\}$. Since $k \geq 2$, the distribution $\mathcal{D}^{1}=\operatorname{span}\left\{g_{1}, h_{2}, a d_{f} g_{1}, a d_{f} h_{2}\right\}$ is involutive, and thus there exists a smooth function $\alpha$ such that $\left[g_{1}, a d_{f} h_{2}\right]=\alpha a d_{f} g_{1} \bmod \operatorname{span}\left\{g_{1}, h_{2}, a d_{f} h_{2}\right\}$. We will show that $\alpha$ is identically zero. According to our assumptions $\mathrm{rk}^{2}=6$ (because $k \geq 2$ and $\mathrm{rk} \mathcal{D}^{k}=2 k+2$ as a direct consequence of the corank assumption cork $\left(\mathcal{D}^{k} \subset \overline{\mathcal{D}}^{k}\right)=2$ ) and, if $\alpha$ is non zero, we would have $\operatorname{rk} \mathcal{D}_{p}^{2}=7$ which is impossible. Hence

$$
\mathcal{D}_{p}^{2}=\operatorname{span}\left\{\frac{\partial}{\partial y_{1}}, \frac{\partial}{\partial y_{2}}, g_{1}, h_{2}, a d_{f} h_{2}, a d_{f}^{2} h_{2}\right\} .
$$

It is easy to prove (by an induction argument) that, for $i \geq 2$,

$$
\mathcal{D}_{p}^{i}=\operatorname{span}\left\{\frac{\partial}{\partial y_{1}}, \frac{\partial}{\partial y_{2}}, g_{1}, \cdots, a d_{f}^{i-2} g_{1}, h_{2}, \cdots, a d_{f}^{i} h_{2}\right\} .
$$

Since the intersection of involutive distributions is an involutive distribution, it follows that $\mathcal{D}_{p}^{i} \cap T X=$ $\operatorname{span}\left\{g_{1}, \cdots, a d_{f}^{i-2} g_{1}, h_{2}, \cdots, a d_{f}^{i} h_{2}\right\}$ is involutive, for $1 \leq i \leq k$. We deduce that

$$
\mathcal{L}^{k}=\operatorname{span}\left\{g_{1}, \cdots, a d_{f}^{k-2} g_{1}, h_{2}, \cdots, a d_{f}^{k} h_{2}\right\}
$$

is involutive (below we will justify denoting this distribution by $\mathcal{L}^{k}$ ). It is immediate that $\mathcal{D}^{k-2} \subset \mathcal{L}^{k} \subset \mathcal{D}^{k}$, where both inclusions are of corank two. The involutivity of $\mathcal{D}_{p}^{k+2}=$ $\operatorname{span}\left\{\frac{\partial}{\partial y_{1}}, g_{1}, \cdots, a d_{f}^{k} g_{1}, h_{2}, \cdots, a d_{f}^{k} h_{2}, a d_{f}^{k+1} h_{2}, a d_{f}^{k+2} h_{2}\right\}$ implies that of $\mathcal{D}^{k}+\operatorname{span}\left\{a d_{f}^{k+1} h_{2}, a d_{f}^{k+2} h_{2}\right\}$ and, since we have supposed $\operatorname{cork}\left(\mathcal{D}^{k} \subset \overline{\mathcal{D}}^{k}\right)=2$, it yields $\overline{\mathcal{D}}^{k}=\mathcal{D}^{k}+\operatorname{span}\left\{a d_{f}^{k+1} h_{2}, a d_{f}^{k+2} h_{2}\right\}$, where $\overline{\mathcal{D}}^{k}$ is the involutive closure of $\mathcal{D}^{k}$.
We show next that $\operatorname{rk}\left(\overline{\mathcal{D}}^{k}+\left[f, \mathcal{D}^{k}\right]\right)=2 k+$ 5. We have $\mathcal{D}_{p}^{k+2}=\operatorname{span}\left\{\frac{\partial}{\partial y_{1}}, \frac{\partial}{\partial y_{2}}\right\}+\overline{\mathcal{D}}^{k}$. Assume $a d_{f}^{k+1} g_{1} \in \overline{\mathcal{D}}^{k}$, if not, the rank in question is, indeed, $2 k+5$. Hence for any vector field $\xi \in \mathcal{D}^{k}$, we have $[f, \xi] \in \overline{\mathcal{D}}^{k}$. By successive applications of the Jacobi identity, it follows immediately that $\overline{\mathcal{D}}^{k}+\left[f, \overline{\mathcal{D}}^{k}\right]=\overline{\mathcal{D}}^{k}$. Therefore, for the prolonged system we obtain

$$
\mathcal{D}_{p}^{k+3}=\operatorname{span}\left\{\frac{\partial}{\partial y_{1}}\right\}+\overline{\mathcal{D}}^{k}+\left[f, \overline{\mathcal{D}}^{k}\right]=\mathcal{D}_{p}^{k+2},
$$

thus contradicting the existence of $\rho$ such that $\operatorname{rk} \mathcal{D}_{p}^{\rho}=$ $n+1$ (recall that $\overline{\mathcal{D}}^{k} \neq T X$ ) and implying that $\Sigma^{(2,0)}$ is not static feedback equivalent to a controllable linear system. By Proposition 1, the system $\Sigma$ would not be flat of differential weight $n+4$ and thus $\operatorname{rk}\left(\overline{\mathcal{D}}^{k}+\right.$ $\left.\left[f, \mathcal{D}^{k}\right]\right)=2 k+5$ and $(A 1)$ holds.

At the beginning of Section III-A, we have shown that $(A 1)$ implies the existence of $g_{2 c} \in \mathcal{D}^{0}$ such that $a d_{f}^{k+1} g_{2 c} \in \overline{\mathcal{D}}^{k}$. We noticed above that $a d_{f}^{k+1} g_{1} \notin \overline{\mathcal{D}}^{k}$, therefore $g_{2 c}=h_{2}$. Furthermore, we have $a d_{f}^{k+2} h_{2} \in \overline{\mathcal{D}}^{k}$, thus $(A 2)$ holds and, moreover, we have $f_{c}=f$, with $f_{c}$ defined by item $(A 3)$. At the beginning of the proof, we have also observed that $\left[g_{1}, h_{2}\right]=0 \bmod \operatorname{span}\left\{h_{2}\right\}$, thus $g_{1 c}=g_{1}$. It follows that

$$
\begin{aligned}
\mathcal{L}^{k} & =\operatorname{span}\left\{a d_{f_{c}}^{q} g_{1 c}, a d_{f_{c}}^{r} g_{2 c}, 0 \leq q \leq k-2,0 \leq r \leq k\right\} \\
& =\operatorname{span}\left\{a d_{f}^{q} g_{1}, a d_{f}^{r} h_{2}, 0 \leq q \leq k-2,0 \leq r \leq k\right\}
\end{aligned}
$$

which justifies denoting the above involutive distribution by $\mathcal{L}^{k}$ and shows $(A 3)$. Recall that $\mathcal{L}^{i}=\mathcal{L}^{i-1}+$ $\left[f_{c}, \mathcal{L}^{i-1}\right]$, for $i \geq k+1$. We thus have

$$
\begin{aligned}
\mathcal{D}_{p}^{k+1} & =\operatorname{span}\left\{\frac{\partial}{\partial y_{1}}, \frac{\partial}{\partial y_{2}}\right\}+\mathcal{L}^{k}+\left[f, \mathcal{L}^{k}\right] \\
& =\operatorname{span}\left\{\frac{\partial}{\partial y_{1}}, \frac{\partial}{\partial y_{2}}\right\}+\mathcal{L}^{k+1}
\end{aligned}
$$

and, by an induction argument,

$$
\mathcal{D}_{p}^{k+i}=\operatorname{span}\left\{\frac{\partial}{\partial y_{1}}, \frac{\partial}{\partial y_{2}}\right\}+\mathcal{L}^{k+i}, i \geq 2 .
$$

Consequently, the involutivity of $\mathcal{D}_{p}^{k+i}$ implies that of $\mathcal{L}^{k+i}$, for $i \geq 1$. Moreover, $\operatorname{rk} \mathcal{D}_{p}^{\rho}=n+2$, implying that $\mathrm{rk} \mathcal{L}^{\rho}=n$, i.e., $\mathcal{L}^{\rho}=T X$, which proves $(A 4)$ and $(A 5)$.

Sufficiency. Consider the control system $\Sigma: \dot{x}=$ $f(x)+u_{1} g_{1}(x)+u_{2} g_{2}(x)$ satisfying $(A 1)-(A 5)$. Transform $\Sigma$ via a static feedback transformation into the form $\tilde{\Sigma}: \dot{x}=f_{c}(x)+\tilde{u}_{1} g_{1 c}(x)+\tilde{u}_{2} g_{2 c}(x)$, where $g_{1 c}, g_{2 c}$ and $f_{c}$ are defined by conditions $(A 1)$ and (A3). By Proposition 5, the involutivity of $\mathcal{L}^{i}=$ $\operatorname{span}\left\{a d_{f}^{q} g_{1}, a d_{f}^{r} h_{2}, 0 \leq q \leq i-2,0 \leq r \leq i\right\}$ follows for $1 \leq i \leq k-1$. It is immediate to see that the prolongation

$$
\Sigma^{(2,0)}:\left\{\begin{array}{l}
\dot{x}=f_{c}(x)+y_{1} g_{1 c}(x)+v_{2} g_{2 c}(x) \\
\dot{y}_{1}=y_{2} \\
\dot{y}_{2}=v_{1}
\end{array}\right.
$$


with $y_{1}=\tilde{u}_{1}$ and $v_{2}=\tilde{u}_{2}$ is locally static feedback linearizable. Indeed, the linearizability distributions $\mathcal{D}_{p}^{i}$, associated to $\Sigma^{(2,0)}$, are of the form

$$
\mathcal{D}_{p}^{i}=\operatorname{span}\left\{\frac{\partial}{\partial y_{1}}, \frac{\partial}{\partial y_{2}}\right\}+\mathcal{L}^{i}, i \geq 1 .
$$

The involutivity of $\mathcal{L}^{i}$ implies that of $\mathcal{D}_{p}^{i}$. Moreover, $\operatorname{rk} \mathcal{L}^{\rho}=n$, thus $\operatorname{rk} D_{p}^{\rho}=n+2$ and $\Sigma^{(2,0)}$ is locally static feedback linearizable. By Proposition 1, the system $\Sigma$ is flat of differential weight $n+4$.

\section{REFERENCES}

[1] R.W. Brockett. Feedback invariants for nonlinear systems. IFAC Congress 6, Helsinki, pages 1115-1120, 1979.

[2] P. Brunovsky. A classification of linear controllable systems. Kybernetika, 3(6):173-188, 1970.

[3] M. Cartan. Sur l'équivalence absolue de certains systèmes d'équations différentielles et sur certaines familles de courbes. Bulletin de la Société mathématique de France, 2(42):12-48, 1914.

[4] B. Charlet, J. Lévine, and R. Marino. Sufficient conditions for dynamic state feedback linearization. SIAM J. Control Optim., 29(1):38-57, 1991.

[5] M. Fliess, J. Levine, P. Martin, and P. Rouchon. Sur les systemes non linéaires différentiellement plats. C. R. Acad. Sci. Paris Sér. I Math., 315(5):619-624, 1992

[6] M. Fliess, J. Lévine, P. Martin, and P. Rouchon. Flatness and defect of non-linear systems: introductory theory and examples. Internat. J. Control, 61(6):1327-1361, 1995.

[7] M. Fliess, J. Lévine, P. Martin, and P. Rouchon. A Lie-Bäcklund approach equivalence and flatness of nonlinear systems. IEEE Trans. Automat. Control, 44(5):922-937, 1999.

[8] D. Hilbert. Über den Begriff der Klasse von Differentialgleichungen. Mathematische Annalen, 73(1):95-108, 1912.

[9] L. Hunt and R. Su. Linear equivalents of nonlinear time varying systems. In Proc. MTNS, Santa Monica, CA, pages 119-123, 1981.

[10] A. Isidori, C.H. Moog, and A. De Luca. A sufficient condition for full linearization via dynamic state feedback. In Proc. IEEE CDC, volume 25, pages 203-208. IEEE, 1986.

[11] B. Jakubczyk. Invariants of dynamic feedback and free systems. In Proc. ECC, pages 1510-1513, 1993.

[12] B. Jakubczyk and W. Respondek. On linearization of control systems. Bull. Acad. Polonaise Sci. Ser. Sci. Math., pages 517-522, 1980.

[13] P. Martin. Contribution à l'étude des systèmes différentiellement plats. PhD thesis, l'Ecole Nationale Supérieure de Mines de Paris, 1992.

[14] P. Martin, P. Rouchon, and R. Murray. Flat systems, equivalence and trajectory generation, CDS Technical Report, Caltech. 2003.

[15] F. Nicolau and W. Respondek. Flatness of multi-input controlaffine systems linearizable via one-fold prolongation. submitted to SIAM Journal on Control and Optimization.

[16] F. Nicolau and W. Respondek. Two-inputs control-affine systems linearizable via one-fold prolongation and their flatness. submitted to European Journal of Control.

[17] F. Nicolau and W. Respondek. Flatness of two-inputs controlaffine systems linearizable via one-fold prolongation. In Proc. IFAC Nolcos, pages 499-504, 2013.

[18] F. Nicolau and W. Respondek. Multi-input control-affine systems linearizable via one-fold prolongation and their flatness. In Proc. IEEE CDC, pages 3249-3254, 2013.

[19] F. Nicolau and W. Respondek. Normal forms for flat controlaffine systems linearizable via one-fold prolongation. In Proc. ECC, pages 2448-2453, 2014.

[20] P.S. Pereira da Silva and C. Corrêa Filho. Relative flatness and flatness of implicit systems. SIAM J. Control Optim., 39(6):19291951, 2001.

[21] J.B. Pomet. A differential geometric setting for dynamic equivalence and dynamic linearization. Banach Center Publ., Vol. 32, pages 319-339, 1995

[22] J.B. Pomet. On dynamic feedback linearization of fourdimensional affine control systems with two inputs. ESAIM Control Optim. Calc. Var, 2:151-230, 1997.
[23] W. Respondek. Symmetries and minimal flat outputs of nonlinear control systems. In New Trends in Nonlinear Dynamics and Control and their Applications, volume LNCIS 295, pages 65-86. Springer, 2003.

[24] K. Schlacher and M. Schoeberl. Construction of flat outputs by reduction and elimination. In Proc. Nolcos 2007, 8(1):666-671, 2007.

[25] M. Van Nieuwstadt, M. Rathinam, and R. Murray. Differential flatness and absolute equivalence of nonlinear control systems. SIAM J. Control Optim., 36(4):1225-1239, 1998. 